\title{
Analysis on the Distribution Channel Strategy of Konka TV
}

\author{
Ya-juan SUN \\ Xijing University, Xi'an, Shaanxi, China
}

\begin{abstract}
Keywords: Distribution Channel Strategy; Konka TV; Problem; Measures.
\end{abstract}
\begin{abstract}
With the development of market economics, distribution channel turns to be an important resource of the enterprise. The distribution channel can bring continuous competitive advantages to the enterprise. In this paper, based on the analysis of the current situation of the distribution channel strategy of Konka TV Station, the main problems existing in the channel strategy are found out by using the methods of literature research, case study and investigation, and targeted improvement measures are put forward, which can gain enlightenment and be used as reference for other related studies. Hopefully, this thesis will contribute a little to making up the theoretical shortage of distribution channel strategy design by practical research.
\end{abstract}

\section{Introduction}

At present, the brand of TV industry is increasing and competition is becoming more and more intense. In order to achieve long-term development and be in a competitive position, it is necessary to find its own shortcomings on the basis of the existing distribution channel strategy, explore and seek optimization and innovation of the channel strategy, so as to realize the cost of the enterprise and achieve the ultimate vision of the enterprise in coping with the times and adversities. Therefore, how to optimize and innovate the distribution channel strategy is the most important task of the enterprise. This paper mainly analyzes the existing problems and causes of Konka TV distribution channel strategy. First of all, the paper studies the background of current TV industry, and studies the distribution channel strategy of Kangka TV by using the methods of literature research, case study and investigation. Secondly, the evolution process of Konka TV distribution channel strategy is studied in depth to analyze current Konka TV distribution channel strategy. Thirdly, through interviews with five senior distributors of Konka TV, combining with the interview results and the actual situation of Konka Company itself, the problems existing in the distribution channel strategy of Konka TV and the reasons for its occurrence have been found. Finally, based on the theoretical knowledge of distribution channel strategy, this paper provides specific measures and ideas for the optimization and innovation of distribution channel strategy, in an attempt to generate reference value for Konka TV in the competitive market environment.

\section{The Current Situation of Konka TV Distribution Channel}

In order to gain a foothold in the fierce competition environment at present and maintain long-term competitive advantage, Konka TV enterprises must establish and develop their core competitiveness, and distribution channels are an important source of building and developing their core competitiveness. With the change of time, it is impossible for any channel to solve the same problem for a long time in the development process of an enterprise. The existing distribution channel strategy should be constantly optimized and innovated according to the change of market environment, so as to adapt the development of society and the progress of the times.

Konka TV is a large composite durable product that needs more sales techniques and after-sales services. Konka group adopts the alternative distribution strategy, and Konka Company chooses several agents or middlemen to sell Konka TV in a certain market. As shown in figure 1, at present, Konka corporation divides the country into six regions, at the same time, it has set up 70 Konka branches, 300 sales and business departments, 200 franchised chain stores and over 3,000 special maintenance outlets, and has cooperated with thousands of authorized dealers at the same time. Second, Konka is directly cooperating with Gome Suning Appliance Chain store. Finally, with the 
development of online retail, Konka Company also supplies products directly to online platforms such as Tmall international supermarket.

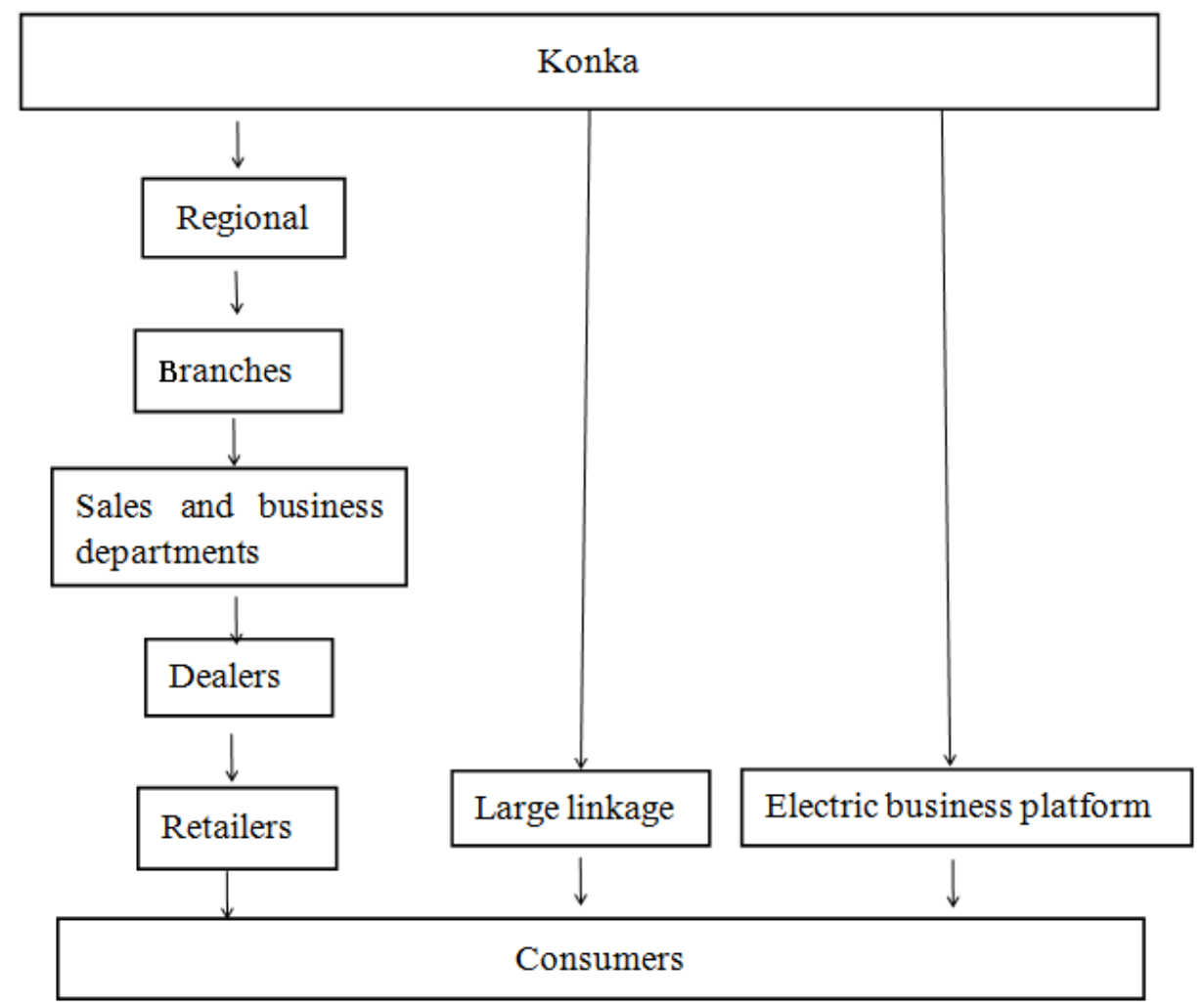

Figure 1. Konka TV distribution channel structure chart

\section{Problems in the Distribution Channel Strategy of Konka TV}

\subsection{High distribution cost}

With the overall home appliance industry slightly depressed, in order to survive in this wave of TV brands, the competition is intensified. Now all major distributors have a lot of brand choices, while Konka TV manufacturers hope that more distributors choose Konka TV and take Konka TV as the main product in the TV area of the store. Therefore, under the background of home appliance industry, dealers begin to hope that Konka appliance manufacturers can provide more profit margins or policy subsidies. In this case, Konka TV group has to make concessions on some interests to motivate distributors to operate Konka TV. Konka TV group chose to subsidize the depreciation of the prototype in exchange for dealers to continue operating Konka TV. There are thousands of Konka authorized dealers across the country, and the samples that are placed in their stores every day wear out. In this case, the distribution costs that Konka faces every day are huge.

\subsection{Channel resource allocation conflicts}

The Internet has had a huge impact on physical store sales. The Internet serves as the medium for producers and end consumers. When conducting shopping, consumers choose to experience goods offline, compare prices with the same goods online, and select the one with price advantage to purchase. Large Internet giant online is the most obvious price advantage, so has serious conflict on the offline sales, destroyed the offline dealer's profit, make the offline sales decline significantly, the dealer for Konka loyalty began to decline, the dealer choices to store or change other brand products, directly affect the customer shopping experience, reduce the brand influence. Second, Gome Suning are in the primary and secondary markets, which have serious conflicts with the third and fourth markets. Value most customers choose to buy what kind of products is the price and quality, now the travel more convenient, three level 4 market customer quantity is very large, many customers choose to Gome Suning to see the price, and then compared with three four level of 
market prices, since most of the product is with three level 4 market price there is a certain gap, therefore has influence on the three level 4 sales, so the conflict also follow.

\subsection{Channel member management is difficult}

In order to reduce costs, improve efficiency and get rid of over-reliance on intermediary channels, Konka appliance manufacturers bypass brokers to set up their own terminal stores or sales outlets locally, manufacturers and sales contact terminals to achieve "zero distance", in order to realize the center of gravity of the channel sink, promote production enterprise channel terminal control and management ability, rapid response to consumer spending habits and consumption preferences, better close to the market, and improve the consumer shopping experience, which is indeed a good idea. However, the current dilemma is the management difficulties of salesmen.

\section{Suggestions to Solve the Problems in Konka TV Distribution Channel Strategy}

\subsection{Display buyer photo album, reduce business distribution cost}

Now the competition of home appliance industry is intense, and each big dealer is the terminal consumer that contacts directly however, the home appliance manufacturer of Konka can choose to give in depreciation cost only, oneself assume depreciation cost or give to the dealer subsidy depreciation cost, bring about cost to be exorbitant Konka TV manufacturer is very hard to promote sale profit again. In addition, with the development of society, consumers are increasingly demanding shopping experience. They not only pursue material enjoyment, but also spiritual satisfaction. The traditional channel on the terminal is limited in size, limiting the decoration space, can only put some popular products with good sales, which cannot meet the personal needs of consumers, thus reducing their shopping experience. Therefore, first of all, the product's performance, core selling point and price should be written on the explosive stickers, so as to attract customers' attention at a glance. Second, Konka TV special after-sale service hangs from door to door or squat down the TV, need to take photo after the installation is complete, then pass all rendering taken to Konka, Konka companies make unified classification and print the image into a photo album to distributors, so that distributors can put the photo album displayed by the buyer in the Konka TV area, give customers more product style choice, reduce the number of prototypes that the distributor sells, in addition to hesitant customers see buyers rendering, sturdy buyers purchase decision, make the customer like in Konka shopping process.

\subsection{Combine online and offline to realize the product model area system}

At present, Konka TV group lags behind in the distribution of channel resources. The following methods can be adopted to reasonably allocate channel resources: the expansion of household appliance online shopping market is a double-edged sword, and only by solving the conflicts and contradictions generated therein can enterprises move to a new height. First, both online and offline channels serve the product, rather than conflict with each other, and finally damage the product. Therefore, it is necessary to combine online and offline products, setting some products as online sales models, some as offline store models, and the last part as a unified online and offline price to eliminate the most critical contradictions. Second, it regularly sends its elite salesmen to hold training sessions to train distributors on the latest retail techniques. The company's elite salesmen tell the dealers about the latest development trend of the industry. Under this trend, consumers' consumption habits and shopping demand changes, and then tell the dealers about the development status of key competitors and the latest products. Finally, the product code system is implemented, and the products sent to each sales area are marked with a unique code, part of the primary and secondary market, the third and fourth level market codes are differentiated, and the product style is slightly changed, making consumers feel that the market is different and the products are different.

\subsection{Grasp internal and external grasp to improve the management level}

In the current rise of two or three line market and rural market, the channels are more dispersed, and the management of salesmen is more difficult. Konka companies can establish mutual trust 
mechanism with the local dealers first. Konka Company regularly organizes product launches, and carries out surgical training and professional management to major dealers at the meetings to form the fate of the manufacturer community. It is of great economic value for the distribution of the Kangka TV channel to make joint efforts to manage the maintenance of the distribution channel and to make it as a means of external promotion channel quality for Konka TV channels of distribution. Second, increase the standards of Konka salesman requests existing Konka salesman in Konka group of internal communication, report the day's sales every day, every month statistics and regional sales to sales is lower than the market as a salesman to punish, higher than the market on sales as salesman for reward, and low cut off part of the sales area of the sales quantity. Finally, regular training sessions were held to promote Konka's corporate culture, narrate Konka's new TV products, analyze recent market trends, and train salesmen's loyalty to Konka as an internal method to improve channel quality.

\subsection{We will strengthen punishment and reward to maintain market order}

The dealer shall be rewarded and punished with clear rewards and penalties. Before selling products with the dealer, a sales contract shall be signed. The contract shall specify that the dealer shall sell products in the reasonable range of unified retail price given by the head office, and severely punish the retailer who changes the use of goods. If unlawful delivery is made to other distributors without the approval of the head office, the supply of television sets shall be stopped or a refund shall be withheld. If the dealer basically meets the requirements of the head office, a certain reward will be given. For a small number of dealers who have maliciously damaged the market environment, they choose to use legal weapons to protect their legitimate rights and interests, and use this case as an example. Finally, the salesmen from different regions are often sent to communicate with salesmen of Konka stores in corresponding regions, and analyze and summarize various customer information and data received from salesmen of Konka stores. In this way, the change of consumer demand is mastered, and it is no longer the top-down guiding of consumers shopping, but the rapid response to market demand, the bottom-up change, and more accurate and professional training for salesmen. The dealer sees the sales person of Konka acute market insight, accurate industry control ability and consummate marketing skill. In this way, distributors will have trust and dependence on Konka and eventually become more loyal to the Konka brand.

\section{Summary}

The competition is very fierce in Chinese household appliance market and it pushes the development and innovation of distribution channel strategy. The marketing of household electrical appliance manufacturers is pushed to transfer from extensive style to intensive style, when the brand, product technique, price and the service trend to be homogenizing. Distribution channel strategy which is an importance element of sales strategy somehow decides if the company could succeed in market environment. In the future development, Konka TV distribution should combined product strategy, channel strategy and promotion strategy together to build a marketing system, to provide support for the future development of Chinese household appliance market distribution.

\section{References}

[1] Shu-hui Sun. Study on optimization of distribution channel strategy of Perfetti Candy Company in Heilongjiang province [D]. Jilin University of Finance and Economics, 2016.

[2] Xuan Zhao, Run H. Niu, Ignacio Castillo. Selecting distribution channel strategies for non-profit organizations [J]. European Journal of Marketing. 2015 (44): 21-23.

[3] Yusen Xia, Tiaojun Xiao, G. Peter Zhang. Distribution Channel Strategies for a Manufacturer with Complementary Products [J]. Decision Sciences. 2012 (44): 46-48.

[4] Kuan-Huei Lee. Timothy Jeonglyeol Lee. Building Channel Strategies and Alliances in Food 
Distribution [J]. Journal of Culinary Science \& Technology. 2013 (7): 41-42.

[5] Junsong Bian, Xiaolei Guo, Kevin W. Li. Distribution channel strategies in a mixed market [J]. International Journal of Production Economics. 2014 (24): 12-15.

[6] Yan-xia Liu. Research on distribution channel management of energy efficiency products of $\mathrm{M}$ company [D]. Beijing Jiaotong University, 2017. 